PROCEEDINGS OF THE

AMERICAN MATHEMATICAL SOCIETY

Volume 133, Number 8, Pages 2309-2317

S 0002-9939(05)08020-2

Article electronically published on March 14, 2005

\title{
SHARPNESS OF THE KORÁNYI APPROACH REGION
}

\author{
KENTARO HIRATA
}

(Communicated by Andreas Seeger)

\begin{abstract}
We prove a Littlewood-type theorem which shows the sharpness of the Korányi approach region for the boundary behavior of Poisson-Szegö integrals on the unit ball of $\mathbb{C}^{n}$. Our result is stronger than Hakim and Sibony (1983).
\end{abstract}

\section{InTRODUCTION}

Let $\mathbb{C}^{n}$ be the $n$-dimensional complex space with inner product

$$
\langle z, w\rangle=\sum_{j=1}^{n} z_{j} \bar{w}_{j}
$$

where $z=\left(z_{1}, \cdots, z_{n}\right)$ and $w=\left(w_{1}, \cdots, w_{n}\right)$, and the associated norm $|z|=$ $\sqrt{\langle z, z\rangle}$. We denote by $B$ the unit ball of $\mathbb{C}^{n}$ and by $S$ its boundary. Let $\sigma$ be the normalized surface measure on $S$. For an integrable function $f$ on $S$, the PoissonSzegö integral of $f$ is defined by

$$
\mathcal{P}[f](z)=\int_{S} \frac{\left(1-|z|^{2}\right)^{n}}{|1-\langle z, \zeta\rangle|^{2 n}} f(\zeta) d \sigma(\zeta) \quad \text { for } z \in B .
$$

In 4], Korányi investigated the boundary behavior of Poisson-Szegö integrals. For $\alpha>1$ and $\xi \in S$, the Korányi approach region at $\xi$ is given by

$$
\mathcal{A}_{\alpha}(\xi)=\left\{z \in B:|1-\langle z, \xi\rangle|<\frac{\alpha}{2}\left(1-|z|^{2}\right)\right\} .
$$

Theorem A. Let $\alpha>1$. If $f$ is an integrable function on $S$, then the PoissonSzegö integral $\mathcal{P}[f](z)$ has the limit $f(\xi)$ as $z \rightarrow \xi$ within $\mathcal{A}_{\alpha}(\xi)$ at almost every point $\xi$ of $S$.

When $n=1$, this theorem is well known as Fatou's theorem. In this case, $\mathcal{A}_{\alpha}(\xi)$ is a non-tangential approach region at $\xi$. The best possibility of this approach region was first proved by Littlewood [5] in the following sense: Let $C_{0}$ be a tangential curve in the unit disc $D$ which ends at $z=1$, and let $C_{\theta}$ be the curve $C_{0}$ rotated about the origin through an angle $\theta$, so that $C_{\theta}$ touches the unit circle internally at $e^{i \theta}$. Then there exists a bounded harmonic function on $D$ which admits no limits as $z \rightarrow e^{i \theta}$ along $C_{\theta}$ for almost every $\theta, 0 \leq \theta \leq 2 \pi$. Aikawa [1] improved this result

Received by the editors September 17, 2003.

2000 Mathematics Subject Classification. Primary 31B25, 31A20, 32A40.

Key words and phrases. Poisson-Szegö integral, Korányi's approach region, boundary behavior.

(C)2005 American Mathematical Society Reverts to public domain 28 years from publication 
by showing that there exists a bounded harmonic function on $D$ which admits no limits as $z \rightarrow e^{i \theta}$ along $C_{\theta}$ for every $\theta$.

In [6], Nagel and Stein proved that the Poisson integral on the upper half space of $\mathbb{R}^{n+1}$ has the boundary limit at almost every point of $\mathbb{R}^{n}$ within a certain approach region which is not contained in any non-tangential approach regions. Sueiro [8] extended Nagel and Stein's result to $\mathbb{C}^{n}$ and proved that the Poisson-Szegö integral has the boundary limit at almost every point of $S$ within a certain approach region which is not contained in any Korányi approach regions.

The purpose of the present paper is to prove a Littlewood-type theorem in higher dimensions. Let $\gamma$ be a curve in $B$ which ends at $e_{1}=(1,0, \cdots, 0)$ and satisfies

$$
\lim _{\substack{z \rightarrow e_{1} \\ z \in \gamma}} \frac{\left|1-\left\langle z, e_{1}\right\rangle\right|}{1-|z|^{2}}=\infty
$$

This means that, for each $\alpha>1$, points of $\gamma$ near $e_{1}$ lie outside $\mathcal{A}_{\alpha}\left(e_{1}\right)$. Let $\mathcal{U}$ denote the group of unitary transformations of $\mathbb{C}^{n}$. We write $U \gamma$ for the image of $\gamma$ through $U \in \mathcal{U}$. Since $U$ preserves inner products, $U \gamma$ touches $S$ internally at $U e_{1}$ and lies outside $\mathcal{A}_{\alpha}\left(U e_{1}\right)$ near $U e_{1}$ for every $\alpha>1$.

Our main result is as follows.

Theorem. Let $\gamma$ be a curve in $B$ which ends at $e_{1}$ and satisfies (1.1). Then there exists a bounded function $f$ on $S$ of which Poisson-Szegö integral $\mathcal{P}[f](z)$ admits no limits as $|z| \rightarrow 1$ along $U \gamma$ for every $U \in \mathcal{U}$, that is,

$$
\liminf _{\substack{|z| \rightarrow 1 \\ z \in U \gamma}} \mathcal{P}[f](z) \neq \limsup _{\substack{|z| \rightarrow 1 \\ z \in U \gamma}} \mathcal{P}[f](z) \quad \text { for every } U \in \mathcal{U} .
$$

Remark 1. Since $\mathcal{U}$ acts transitively on $S$, for each $\xi \in S$ there is $U_{\xi} \in \mathcal{U}$ such that $\xi=U_{\xi} e_{1}$. Therefore, the Theorem implies that there exists a bounded PoissonSzegö integral which admits no limits as $z \rightarrow \xi$ along $U_{\xi} \gamma$ at every point $\xi$ of $S$. Moreover, we can make $f$ satisfy

$$
\liminf _{\substack{|z| \rightarrow 1 \\ z \in U \gamma}} \mathcal{P}[f](z)=\inf _{\zeta \in S} f(\zeta) \text { and } \limsup _{\substack{|z| \rightarrow 1 \\ z \in U \gamma}} \mathcal{P}[f](z)=\sup _{\zeta \in S} f(\zeta)
$$

for every $U \in \mathcal{U}$.

Remark 2. By Sueiro's result, the limit in (1.1) cannot be replaced by the upper limit.

As a related topic in higher dimensions, there is the following result due to Hakim and Sibony [3.

Theorem B. Suppose $n>1$. Let $\alpha>1$ and $h:(0,1] \rightarrow[\alpha, \infty)$ be a decreasing function such that

$$
\lim _{x \rightarrow 0+} h(x)=\infty
$$

and let

$$
D_{\alpha, h}(\xi)=\left\{z \in B: \begin{array}{l}
|1-\langle z, \xi\rangle| \leq \alpha(1-|\langle z, \xi\rangle|) \text { and } \\
|1-\langle z, \xi\rangle| \leq h(|1-\langle z, \xi\rangle|)(1-|z|)
\end{array}\right\} .
$$

Then there exists a bounded holomorphic function on $B$ which admits no limits as $z \rightarrow \xi$ within $D_{\alpha, h}(\xi)$ at almost every point $\xi$ of $S$. 
We note that the approach region $D_{\alpha, h}(\xi)$ is wider than any Korányi approach regions in the complex tangential directions, but is the same in the special real direction. Our theorem is stronger than Theorem B in the following points:

- It improves no convergence "almost everywhere" to "everywhere".

- It establishes that a tangential approach in the special real direction can not be allowed in Theorem A.

- The existence of a bounded Poisson-Szegö integral which fails to have a boundary limit is ensured even if we replace $D_{\alpha, h}\left(e_{1}\right)$ by much smaller curve $\gamma$ satisfying (1.1).

Also, our method is different from Hakim and Sibony's. Theorem B is proved by constructing a higher-dimensional Blaschke product. However, we will prove the Theorem in Section 3 by constructing a bounded function on $S$ and using lower and upper estimates of Poisson-Szegö integrals in Section 2. In the proofs we adapt ideas from 1, 2. Whereas the polar and the euclidean coordinates were used to construct a bounded function on the unit circle and on $\mathbb{R}^{n}$ in 1 , 2, they are not applicable in our case. This is an important difference between [1, 2] and our case.

Throughout the paper we use the symbols $A_{0}, A_{1}, A_{2}, \cdots$ to denote absolute positive constants depending only on the dimension $n$.

\section{Estimates of Poisson-Szegö integrals}

In this section we give lower and upper estimates for Poisson-Szegö integrals. To this end, we start by introducing a non-isotropic ball in $S$. We observe that the function $d(z, w)=|1-\langle z, w\rangle|^{1 / 2}$ satisfies the triangle inequality on $B \cup S$, and defines a metric on $S$. See [7, Lemma 7.3]. For $\xi \in S$ and $r>0$, we write

$$
Q(\xi, r)=\{\zeta \in S: d(\zeta, \xi)<r\}
$$

the non-isotropic ball of center $\xi$ and radius $r$. Note that, to emphasize the metric $d$, we use the slightly different definition from Stoll's book. We observe that

$$
\sigma(Q(U \xi, r))=\sigma(Q(\xi, r))
$$

for any unitary transformations $U$ and that

$$
\lim _{r \rightarrow 0} \frac{\sigma(Q(\xi, r))}{r^{2 n}}=\frac{2^{n}}{4 \sqrt{\pi}} \frac{\Gamma\left(\frac{n+1}{2}\right)}{\Gamma\left(\frac{n}{2}+1\right)}
$$

See [7. p. 84]. Moreover, there is a constant $A_{0}>1$ depending only on the dimension $n$ such that

$$
A_{0}^{-1} r^{2 n} \leq \sigma(Q(\xi, r)) \leq A_{0} r^{2 n}
$$

for $\xi \in S$ and $0 \leq r \leq \operatorname{diam} S=\sqrt{2}$. Here $\operatorname{diam} F=\sup \{d(\eta, \zeta): \eta, \zeta \in F\}$ for $F \subset S$.

Let $T>0$ and $\xi \in S$. For an integrable function $g$ on $S$, we define the truncated maximal function at $\xi$ by

$$
\mathcal{M}_{T}[g](\xi)=\sup _{r \geq T} r^{-2 n} \int_{Q(\xi, r)}|g(\zeta)| d \sigma(\zeta) .
$$

By the argument in [7, Theorem 7.8], we obtain the following estimate for the Poisson-Szegö integral. For completeness we give the proof. 
Lemma 1. There exists a positive constant $A_{1}$ depending only on the dimension $n$ such that if $g$ is an integrable function on $S$ and $C>0$, then

$$
|\mathcal{P}[g](t \xi)| \leq A_{1}\left((1-t)^{-n} \int_{Q(\xi, C \sqrt{1-t})}|g(\zeta)| d \sigma(\zeta)+C^{-2 n} \mathcal{M}_{C \sqrt{1-t}}[g](\xi)\right)
$$

for $\xi \in S$ and $0<t<1$.

Proof. Let $\xi \in S$ and $0<t<1$ be fixed, and let

$$
\begin{aligned}
& V_{0}=Q(\xi, C \sqrt{1-t}), \\
& V_{j}=Q\left(\xi, 2^{j} C \sqrt{1-t}\right) \backslash Q\left(\xi, 2^{j-1} C \sqrt{1-t}\right) \quad(j=1, \cdots, N),
\end{aligned}
$$

where $N$ is the smallest integer such that $2^{N} C \sqrt{1-t}>\sqrt{2}$. Then

$$
|\mathcal{P}[g](t \xi)| \leq \sum_{j=0}^{N} \int_{V_{j}} \frac{\left(1-t^{2}\right)^{n}}{|1-\langle t \xi, \zeta\rangle|^{2 n}}|g(\zeta)| d \sigma(\zeta) .
$$

Since $|1-\langle t \xi, \zeta\rangle| \geq 1-t$ for $\zeta \in S$, it follows that

$$
\int_{V_{0}} \frac{\left(1-t^{2}\right)^{n}}{|1-\langle t \xi, \zeta\rangle|^{2 n}}|g(\zeta)| d \sigma(\zeta) \leq \frac{2^{n}}{(1-t)^{n}} \int_{Q(\xi, C \sqrt{1-t})}|g(\zeta)| d \sigma(\zeta)
$$

Let $j=1, \cdots, N$. By the triangle inequality, we have for $\zeta \in V_{j}$,

$$
2^{j-1} C \sqrt{1-t} \leq d(\xi, \zeta) \leq d(\xi, t \xi)+d(t \xi, \zeta) \leq 2 d(t \xi, \zeta)=2|1-\langle t \xi, \zeta\rangle|^{1 / 2} .
$$

Hence it follows that

$$
\begin{aligned}
\int_{V_{j}} \frac{\left(1-t^{2}\right)^{n}}{11-\left.\langle t \xi, \zeta\rangle\right|^{2 n}}|g(\zeta)| d \sigma(\zeta) & \leq \frac{2^{9 n}}{2^{4 n j} C^{4 n}(1-t)^{n}} \int_{Q\left(\xi, 2^{j} C \sqrt{1-t}\right)}|g(\zeta)| d \sigma(\zeta) \\
& \leq \frac{2^{9 n}}{2^{2 n j} C^{2 n}} \mathcal{M}_{C \sqrt{1-t}}[g](\xi) .
\end{aligned}
$$

Noting that $\sum_{j=1}^{N} 2^{-2 n j}<1$, we obtain the lemma with $A_{1}=2^{9 n}$.

As a consequence of Lemma 1, we obtain the following upper and lower estimates.

Lemma 2. The following statements hold:

(i) If $g$ is an integrable function on $S$, then

$$
|\mathcal{P}[g](t \xi)| \leq A_{2} \mathcal{M}_{\sqrt{1-t}}[g](\xi) \quad \text { for } \xi \in S \text { and } 0<t<1,
$$

where $A_{2}$ is a positive constant depending only on the dimension $n$.

(ii) Let $\xi \in S, 0<r<1$ and $C>0$. If $g$ is a measurable function on $S$ such that $g=1$ on $Q(\xi, C \sqrt{1-r})$ and $|g| \leq 1$ on $S$, then

$$
\mathcal{P}[g](t \xi) \geq 1-\frac{A_{3}}{C^{2 n}} \quad \text { for } r \leq t<1,
$$

where $A_{3}$ is a positive constant depending only on the dimension $n$.

Proof. Putting $C=1$ in Lemma 1 , we obtain (ii) with $A_{2}=2 A_{1}$. Let us show (ii). We put $h=(1-g) / 2$. Then $h=0$ on $Q(\xi, C \sqrt{1-r})$ and $|h| \leq 1$ on $S$. Applying Lemma 1 to $h$, we obtain from (2.2) that for $r \leq t<1$,

$$
\mathcal{P}[h](t \xi) \leq \frac{A_{1}}{C^{2 n}} \mathcal{M}_{C \sqrt{1-t}}[h](\xi) \leq \frac{A_{1}}{C^{2 n}} \sup _{\rho \geq C \sqrt{1-t}} \frac{\sigma(Q(\xi, \rho))}{\rho^{2 n}} \leq \frac{A_{0} A_{1}}{C^{2 n}} .
$$

Since $\mathcal{P}[g]=1-2 \mathcal{P}[h]$, we obtain (1ii) with $A_{3}=2 A_{0} A_{1}$. 


\section{Proof of the Theorem}

Let $\pi$ be the radial projection to $S$ defined by $\pi(z)=z /|z|$ for $z \neq 0$. We note that (1.1) implies

$$
\lim _{\substack{z \rightarrow e_{1} \\ z \in \gamma}} \frac{d\left(z, e_{1}\right)}{d(z, \pi(z))}=\infty
$$

since $1-|z|^{2} \geq 1-|z|=d(z, \pi(z))^{2}$ for $z \in B \backslash\{0\}$. Recall that

$$
\operatorname{diam} F=\sup _{\eta, \zeta \in F} d(\eta, \zeta) \quad \text { for } F \subset S .
$$

Lemma 3. Let $\gamma$ be the curve as in the Theorem. Then there exist sequences of positive numbers $\left\{a_{j}\right\}_{j=1}^{\infty},\left\{b_{j}\right\}_{j=1}^{\infty}$ and subcurves $\left\{\gamma_{j}\right\}_{j=1}^{\infty}$ of $\gamma$ with the following properties:

(i) $0<a_{j}<b_{j}<a_{j+1}<b_{j+1}<1$ and $\lim _{j \rightarrow \infty} a_{j}=1$;

(ii) $a_{j} \leq|z| \leq b_{j}$ for $z \in \gamma_{j}$;

(iii) $\operatorname{diam} \pi\left(\gamma_{j}\right) \leq \sqrt{1-b_{j-1}}$ if $j \geq 2$;

(iv) $\lim _{j \rightarrow \infty} \frac{\operatorname{diam} \pi\left(\gamma_{j}\right)}{\sqrt{1-a_{j}}}=\infty$.

Proof. Let $\alpha_{j}>1$ be such that $\alpha_{j} \rightarrow \infty$ as $j \rightarrow \infty$. We shall choose $\left\{a_{j}\right\},\left\{b_{j}\right\}$ and $\left\{\gamma_{j}\right\}$, inductively. By (3.1), we can find $a_{1}$ with $\inf _{z \in \gamma}|z|<a_{1}<1$ and

$$
d\left(z, e_{1}\right) \geq \alpha_{1} d(z, \pi(z)) \quad \text { for } z \in \gamma \cap\left\{|z| \geq a_{1}\right\} .
$$

Let $\gamma^{\prime}$ be the connected component of $\gamma \cap\left\{|z| \geq a_{1}\right\}$ which ends at $e_{1}$. Since there is $z_{0} \in \gamma^{\prime} \cap\left\{|z|=a_{1}\right\}$, we have from the triangle inequality that

$$
\begin{aligned}
\operatorname{diam} \pi\left(\gamma^{\prime}\right) & \geq d\left(\pi\left(z_{0}\right), e_{1}\right) \\
& \geq d\left(z_{0}, e_{1}\right)-d\left(z_{0}, \pi\left(z_{0}\right)\right) \\
& \geq\left(\alpha_{1}-1\right) d\left(z_{0}, \pi\left(z_{0}\right)\right) \\
& =\left(\alpha_{1}-1\right) \sqrt{1-a_{1}} .
\end{aligned}
$$

Let $\gamma^{\prime \prime}$ be a subcurve of $\gamma^{\prime}$ connecting a point in $\left\{|z|=a_{1}\right\}$ and a point near $e_{1}$ such that

$$
\operatorname{diam} \pi\left(\gamma^{\prime \prime}\right) \geq \frac{1}{2} \operatorname{diam} \pi\left(\gamma^{\prime}\right)
$$

We take $b_{1}$ so that $\sup _{z \in \gamma^{\prime \prime}}|z|<b_{1}<1$, and let $\gamma_{1}$ be the connected component of $\gamma \cap\left\{a_{1} \leq|z| \leq b_{1}\right\}$ containing $\gamma^{\prime \prime}$. Then

$$
\operatorname{diam} \pi\left(\gamma_{1}\right) \geq \operatorname{diam} \pi\left(\gamma^{\prime \prime}\right) \geq \frac{\alpha_{1}-1}{2} \sqrt{1-a_{1}} .
$$

We next choose $a_{2}, b_{2}$ and $\gamma_{2}$ as follows. Let $a_{2}$ be such that $b_{1}<a_{2}<1$ and

$$
\frac{1}{4} \sqrt{1-b_{1}} \geq d\left(z, e_{1}\right) \geq \alpha_{2} d(z, \pi(z)) \quad \text { for } z \in \gamma \cap\left\{|z| \geq a_{2}\right\}
$$

By repeating the above procedure, we can find $b_{2}$ and $\gamma_{2}$ with $a_{2}<b_{2}<1$ and $a_{2} \leq|z| \leq b_{2}$ for $z \in \gamma_{2}$, and

$$
\operatorname{diam} \pi\left(\gamma_{2}\right) \geq \frac{\alpha_{2}-1}{2} \sqrt{1-a_{2}}
$$


It also follows from (3.2) and $\alpha_{2}>1$ that

$$
d\left(\pi(z), e_{1}\right) \leq d\left(z, e_{1}\right)+d(z, \pi(z)) \leq \frac{1}{2} \sqrt{1-b_{1}} \quad \text { for } z \in \gamma_{2},
$$

and so diam $\pi\left(\gamma_{2}\right) \leq \sqrt{1-b_{1}}$. Hence $\gamma_{2}$ satisfies (iii). Continuing this procedure, we obtain the required sequences.

In the rest of this section, we suppose that $\left\{a_{j}\right\},\left\{b_{j}\right\}$ and $\left\{\gamma_{j}\right\}$ are as in Lemma 3) and put

$$
\ell_{j}=\frac{\operatorname{diam} \pi\left(\gamma_{j}\right)}{4}, \quad c_{j}=\left(\frac{\operatorname{diam} \pi\left(\gamma_{j}\right)}{\sqrt{1-a_{j}}}\right)^{1 / 2} \quad \text { and } \quad \rho_{j}=c_{j} \sqrt{1-a_{j}}
$$

to simplify the notation. Note from Lemma 3 that

$$
\lim _{j \rightarrow \infty} \ell_{j}=0, \quad \lim _{j \rightarrow \infty} \frac{\rho_{j}}{\ell_{j}}=0 \quad \text { and } \quad \lim _{j \rightarrow \infty} c_{j}=\infty .
$$

Therefore, in the argument below, we may assume that $\rho_{j}<\ell_{j}$ for every $j \in \mathbb{N}$.

For each $j \in \mathbb{N}$, let us choose finitely many points $\left\{\eta_{j}^{\nu}\right\}_{\nu}$ in $S$ such that

(P1) $S=\bigcup_{\nu} Q\left(\eta_{j}^{\nu}, \ell_{j}\right)$,

(P2) $\left\{Q\left(\eta_{j}^{\nu}, \ell_{j} / 2\right)\right\}_{\nu}$ are mutually disjoint.

This is possible. In fact, we first take an arbitrary $\eta_{j}^{1} \in S$, and take $\eta_{j}^{\mu} \in S \backslash$ $\bigcup_{\nu=1}^{\mu-1} Q\left(\eta_{j}^{\nu}, \ell_{j}\right)$ inductively as long as $S \backslash \bigcup_{\nu=1}^{\mu-1} Q\left(\eta_{j}^{\nu}, \ell_{j}\right) \neq \emptyset$. Since $S$ is compact, we can get finitely many points $\left\{\eta_{j}^{\nu}\right\}_{\nu}$ satisfying (P1). It also fulfills that $d\left(\eta_{j}^{\nu}, \eta_{j}^{\mu}\right) \geq \ell_{j}$ if $\nu \neq \mu$ by the definition of the non-isotropic ball. Hence (P2) follows from the triangle inequality.

We put

$$
M_{j}=\bigcup_{\nu}\left\{\zeta \in S: d\left(\zeta, \eta_{j}^{\nu}\right)=\ell_{j}\right\} .
$$

Then $\pi\left(U \gamma_{j}\right) \cap M_{j} \neq \emptyset$ for any unitary transformations $U$. In fact, there is $\nu$ such that $\pi\left(U \gamma_{j}\right) \cap Q\left(\eta_{j}^{\nu}, \ell_{j}\right) \neq \emptyset$ by (P1). Since $\operatorname{diam} \pi\left(U \gamma_{j}\right)=\operatorname{diam} \pi\left(\gamma_{j}\right)=4 \ell_{j}$ and $\operatorname{diam} Q\left(\eta_{j}^{\nu}, \ell_{j}\right) \leq 2 \ell_{j}$, we have $\pi\left(U \gamma_{j}\right) \cap\left\{\zeta \in S: d\left(\zeta, \eta_{j}^{\nu}\right)=\ell_{j}\right\} \neq \emptyset$, and so $\pi\left(U \gamma_{j}\right) \cap M_{j} \neq \emptyset$. Let $G_{j}$ be the subset of $B$ given by

$$
G_{j}=\left\{z \in B: a_{j} \leq|z| \leq b_{j} \text { and } \pi(z) \in M_{j}\right\} .
$$

Since $U \gamma_{j} \subset\left\{a_{j} \leq|z| \leq b_{j}\right\}$ by Lemma 3 (iii), it follows that $U \gamma_{j} \cap G_{j} \neq \emptyset$. We also put

$$
E_{j}=\bigcup_{\nu} R_{j}^{\nu},
$$

where $R_{j}^{\nu}=\left\{\zeta \in S: \ell_{j}-\rho_{j}<d\left(\zeta, \eta_{j}^{\nu}\right)<\ell_{j}+\rho_{j}\right\}$ is the non-isotropic ring. Since the value $\sigma\left(R_{j}^{\nu}\right)$ is independent of $\eta_{j}^{\nu}$ by unitary invariance, we write $\kappa_{j}$ for this value. We note that

$$
\lim _{j \rightarrow \infty} \frac{\kappa_{j}}{\ell_{j}^{2 n}}=0
$$


In fact, we obtain from (2.1) and (3.3) that for $\eta \in S$,

$$
\begin{aligned}
\frac{\kappa_{j}}{\ell_{j}^{2 n}} & =\frac{\sigma\left(Q\left(\eta, \ell_{j}+\rho_{j}\right)\right)-\sigma\left(Q\left(\eta, \ell_{j}-\rho_{j}\right)\right)}{\ell_{j}^{2 n}} \\
& =\left(\frac{\ell_{j}+\rho_{j}}{\ell_{j}}\right)^{2 n} \frac{\sigma\left(Q\left(\eta, \ell_{j}+\rho_{j}\right)\right)}{\left(\ell_{j}+\rho_{j}\right)^{2 n}}-\left(\frac{\ell_{j}-\rho_{j}}{\ell_{j}}\right)^{2 n} \frac{\sigma\left(Q\left(\eta, \ell_{j}-\rho_{j}\right)\right)}{\left(\ell_{j}-\rho_{j}\right)^{2 n}} \\
& \longrightarrow 0 \quad \text { as } j \rightarrow \infty .
\end{aligned}
$$

Lemma 4. Let $\left\{E_{j}\right\}$ be as above, and let $\chi_{E_{j}}$ denote the characteristic function of $E_{j}$. Then the following properties hold:

(i) $\lim _{j \rightarrow \infty}\left(\sup _{|z| \leq b_{j-1}} \mathcal{P}\left[\chi_{E_{j}}\right](z)\right)=0$.

(ii) $\lim _{j \rightarrow \infty} \sigma\left(E_{j}\right)=0$.

Proof. Let $z \in B$ be such that $|z| \leq b_{j-1}$. By Lemma 2(ii), we have

$$
\begin{aligned}
\mathcal{P}\left[\chi_{E_{j}}\right](z) & \leq A_{2} \mathcal{M}_{\sqrt{1-|z|}}\left[\chi_{E_{j}}\right](\pi(z)) \\
& \leq A_{2} \sup _{r \geq \sqrt{1-|z|}} r^{-2 n} \sum_{\nu} \sigma\left(R_{j}^{\nu} \cap Q(\pi(z), r)\right) \\
& \leq A_{2} \sup _{r \geq \sqrt{1-|z|}} r^{-2 n} N_{j}(z, r) \kappa_{j},
\end{aligned}
$$

where $N_{j}(z, r)$ is the number of $\eta_{j}^{\nu}$ such that $R_{j}^{\nu} \cap Q(\pi(z), r) \neq \emptyset$. Since $\sqrt{1-|z|} \geq$ $\operatorname{diam} \pi\left(\gamma_{j}\right)$ by Lemma3(iii), we observe from $\rho_{j}<\ell_{j} \leq r / 4$ that if $R_{j}^{\nu} \cap Q(\pi(z), r) \neq$ $\emptyset$, then $Q\left(\eta_{j}^{\nu}, \ell_{j} / 2\right) \subset Q(\pi(z), 2 r)$. Therefore it follows from (2.2) and (P2) that $N_{j}(z, r) \leq A_{4}\left(r / \ell_{j}\right)^{2 n}$ with a positive constant $A_{4}$ depending only on the dimension $n$. Hence we obtain

$$
\mathcal{P}\left[\chi_{E_{j}}\right](z) \leq A_{2} A_{4} \frac{\kappa_{j}}{\ell_{j}^{2 n}},
$$

so that (i) follows from (3.4).

Taking $z=0$ in (i), we obtain

$$
\sigma\left(E_{j}\right)=\mathcal{P}\left[\chi_{E_{j}}\right](0) \longrightarrow 0 \quad \text { as } j \rightarrow \infty .
$$

Thus (iii) follows.

We now construct a bounded function $f$ on $S$ satisfying the property in the Theorem.

Proof of the Theorem. In view of Lemma 4, taking a subsequence of $j$ if necessary, we may assume that

$$
\mathcal{P}\left[\chi_{E_{j}}\right](z) \leq 2^{-j} \quad \text { for }|z| \leq b_{j-1},
$$

and $\sigma\left(E_{j}\right) \leq 2^{-j}$. Then $\sigma\left(\bigcap_{k} \bigcup_{j=k}^{\infty} E_{j}\right)=0$. Let

$$
f_{j}(\zeta)= \begin{cases}(-1)^{I_{j}(\zeta)} & \text { if } \zeta \in \bigcup_{i=1}^{j} E_{i}, \\ 0 & \text { if } \zeta \notin \bigcup_{i=1}^{j} E_{i},\end{cases}
$$


where $I_{j}(\zeta)$ is the maximum integer $i$ such that $\zeta \in E_{i}$ for $\zeta \in \bigcup_{i=1}^{j} E_{i}$. Then we observe that $f_{j}$ converges almost everywhere on $S$ to

$$
f(\zeta)= \begin{cases}(-1)^{I(\zeta)} & \text { if } \zeta \in \bigcup_{j=1}^{\infty} E_{j} \backslash \bigcap_{k} \bigcup_{j=k}^{\infty} E_{j}, \\ 0 & \text { if } \zeta \notin \bigcup_{j=1}^{\infty} E_{j} \text { or } \zeta \in \bigcap_{k} \bigcup_{j=k}^{\infty} E_{j},\end{cases}
$$

where $I(\zeta)$ is the maximum integer $i$ such that $\zeta \in E_{i}$ for $\zeta \in \bigcup_{j=1}^{\infty} E_{j} \backslash \bigcap_{k} \bigcup_{j=k}^{\infty} E_{j}$. We also see that

(a) $f_{j}=(-1)^{j}$ on $E_{j}$ and $\left|f_{j}\right| \leq 1$ on $S$,

(b) $\left|f_{j+1}-f_{j}\right| \leq 2 \chi_{E_{j+1}}$

(c) $\mathcal{P}\left[f_{j}\right]$ converges to $\mathcal{P}[f]$ on $B$.

Let $U$ be a unitary transformation. Since $U \gamma$ intersects $G_{j}$ for every $j$ as stated in the paragraph defining $G_{j}$, we can take $z_{j} \in U \gamma \cap G_{j}$. Note that $a_{j} \leq\left|z_{j}\right| \leq b_{j}$ and $Q\left(\pi\left(z_{j}\right), c_{j} \sqrt{1-a_{j}}\right) \subset E_{j}$. If $j$ is even, then it follows from Lemma 2)(1ii), Lemma 3(i) and (3.5) that

$$
\begin{aligned}
\mathcal{P}[f]\left(z_{j}\right) & =\mathcal{P}\left[f_{j}\right]\left(z_{j}\right)+\sum_{k=j}^{\infty} \mathcal{P}\left[f_{k+1}-f_{k}\right]\left(z_{j}\right) \\
& \geq \mathcal{P}\left[f_{j}\right]\left(z_{j}\right)-\sum_{k=j}^{\infty} \mathcal{P}\left[\left|f_{k+1}-f_{k}\right|\right]\left(z_{j}\right) \\
& \geq 1-\frac{A_{3}}{c_{j}^{2 n}}-2 \sum_{k=j}^{\infty} \mathcal{P}\left[\chi_{E_{k+1}}\right]\left(z_{j}\right) \\
& \geq 1-\frac{A_{3}}{c_{j}^{2 n}}-2 \sum_{k=j}^{\infty} 2^{-k-1} \\
& =1-\frac{A_{3}}{c_{j}^{2 n}}-2^{1-j}
\end{aligned}
$$

Similarly, if $j$ is odd, then

$$
\mathcal{P}[f]\left(z_{j}\right) \leq-1+\frac{A_{3}}{c_{j}^{2 n}}+2^{1-j} .
$$

Hence we obtain

$$
\liminf _{\substack{|z| \rightarrow 1 \\ z \in U \gamma}} \mathcal{P}[f](z)=-1<1=\limsup _{\substack{|z| \rightarrow 1 \\ z \in U \gamma}} \mathcal{P}[f](z)
$$

by (3.3). Thus the Theorem is proved.

\section{ACKNOWLEDGMENT}

The author expresses his deep gratitude to Professor Hiroaki Aikawa for his valuable advice and encouragement.

\section{REFERENCES}

1. H. Aikawa, Harmonic functions having no tangential limits, Proc. Amer. Math. Soc. 108 (1990), no. 2, 457-464. MR0990410 (90h:31003)

2. Harmonic functions and Green potentials having no tangential limits, J. London Math. Soc. (2), 43 (1991), no. 1, 125-136. MR1099092 (92b:31005) 
3. M. Hakim and N. Sibony, Fonctions holomorphes bornées et limites tangentielles, Duke Math. J. 50 (1983), no. 1, 133-141. MR0700133 (84m:32011)

4. A. Korányi, Harmonic functions on Hermitian hyperbolic space, Trans. Amer. Math. Soc. 135 (1969), 507-516. MR0277747 (43:3480)

5. J. E. Littlewood, On a theorem of Fatou, J. London Math. Soc. 2 (1927), 172-176.

6. A. Nagel and E. M. Stein, On certain maximal functions and approach regions, Adv. in Math. 54 (1984), 83-106. MR0761764 (86a:42026)

7. M. Stoll, Invariant potential theory in the unit ball of $C^{n}$, London Mathematical Society Lecture Note Series, 199. Cambridge University Press, Cambridge, 1994. MR.1297545 (96f:31011)

8. J. Sueiro, On maximal functions and Poisson-Szegö integrals, Trans. Amer. Math. Soc. 298 (1986), no. 2, 653-669. MR0860386 (87m:42017)

Department of Mathematics, Shimane University, Matsue 690-8504, Japan

E-mail address: hirata@math.shimane-u.ac.jp 